\title{
Clinical Outcomes and Histological Patterns in Oxalate Nephropathy due to Enteric and Nonenteric Risk Factors
}

\author{
Swetha Reddy ${ }^{a}$ Erin Bolen ${ }^{b}$ Mina Abdelmalek ${ }^{a} \quad$ John C. Lieske ${ }^{c}$ \\ Maggie Ryan ${ }^{d}$ Mira T. Keddis ${ }^{a}$ \\ aDivision of Nephrology, Department of Medicine, Mayo Clinic, Scottsdale, AZ, USA; bMayo Clinic Alix School \\ of Medicine, Mayo Clinic, Scottsdale, AZ, USA; 'Division of Nephrology, Department of Medicine, Mayo Clinic, \\ Rochester, MN, USA; ${ }^{d}$ Department of Pathology, Mayo Clinic, Scottsdale, AZ, USA
}

\section{Keywords}

Oxalate nephropathy · Enteric hyperoxaluria $\cdot$ Vitamin C

\begin{abstract}
Introduction: Current knowledge of risk factors and renal histologic patterns of oxalate nephropathy $(\mathrm{ON})$ not due to primary hyperoxaluria $(\mathrm{PH})$ has been limited to small case series and case reports. Thus, we analyzed and compared clinical risk factors, histologic characteristics, and renal outcomes of patients with biopsy-confirmed ON among a cohort of patients with enteric and nonenteric risk factors. Methods: A clinical data repository of native kidney pathology reports from 2009 to 2020 at all Mayo Clinic sites was used to identify 421 ON cases. Results: After excluding cases in transplanted kidneys or due to $\mathrm{PH}, 64$ cases remained. Enteric risk factors were present in 30 and nonenteric in 34. Roux-en-Y gastric bypass (17) and pancreatic insufficiency (6) were most common in the enteric hyperoxaluria group. In the nonenteric group, vitamin C (7) and dietary oxalate (7) were common, while no apparent risk was noted in 16 . Acute kidney injury (AKI) stage III at the time of diagnosis was present in $60 \%$, and $40.6 \%$ required dialysis. Patients in the non-
\end{abstract}

enteric group had more interstitial inflammation ( $p=0.01$ ), and a greater number of tubules contained intratubular calcium oxalate $(\mathrm{CaOx})$ crystals $(p=0.001)$ than the nonenteric group. Patients in the enteric group were more likely to have baseline chronic kidney disease (CKD) $(p=0.02)$ and moderate-to-severe tubulointerstitial fibrosis and atrophy (IFTA) (OR 3.49, $p=0.02$ ). After a median follow-up of 10 months, $39 \%$ were dialysis dependent, $11 \%$ received a kidney transplant, and $32 \%$ died. On univariate analysis, $>10$ tubules with $\mathrm{CaOx}$ crystals, baseline CKD, and AKI requiring dialysis correlated with the risk of dialysis, transplant, or death. On multivariate analysis, only AKI requiring dialysis correlated with adverse renal outcomes. Conclusion: This is the largest cohort study of ON not due to $\mathrm{PH}$. Histologic features differ in patients with enteric versus nonenteric risks. Patients in the enteric group are more likely to have baseline CKD and significant IFTA, while patients in the nonenteric group were more likely to have a greater number of tubules with $\mathrm{CaOx}$ crystals and corresponding interstitial inflammation. AKI requiring dialysis at the time of diagnosis was the single most significant predictor of adverse renal outcome.

(c) 2021 S. Karger AG, Basel karger@karger.com

www.karger.com/ajn

Karger\%$$
\text { r }
$$

(C) 2021 S. Karger AG, Basel
Correspondence to:

Mira T. Keddis, keddis.mira@ mayo.edu 


\section{Introduction}

Oxalate nephropathy $(\mathrm{ON})$ is defined as calcium oxalate $(\mathrm{CaOx})$ crystal deposition within the renal tubulointerstitial compartment with impaired kidney function. Two main clinical contexts have been associated with $\mathrm{ON}$ : primary hyperoxaluria $(\mathrm{PH})$ due to inborn errors of glyoxylate metabolism and secondary hyperoxaluria due to enteric causes or high dietary oxalate content. Enteric hyperoxaluria $(\mathrm{EH})$ is a state of increased intestinal absorption of dietary oxalate due to a wide spectrum of gastrointestinal pathophysiologic processes associated with fat malabsorption and increased gut permeability [1-3]. ON was first linked to EH in 1975 after small intestinal bypass surgery [4]. In 2001, Mole et al. [5] presented a case series of 8 patients with ON secondary to jejunoileal bypass, reviewed 18 case reports in the literature, and highlighted that ON was often underreported and could occur $>10$ years after malabsorptive surgery [5]. In 2008, Nasr et al. [6] reported on 11 cases of ON after Roux-enY gastric bypass (RYGB) with dire renal outcomes in 8 patients including chronic kidney disease (CKD) in all and kidney failure in 3 after a median follow-up of 1 year. In 2011, Cartery et al. [7] reported on 12 cases of ON secondary to chronic pancreatitis and reported comparatively better renal outcomes of 3 patients with kidney failure and 8 with CKD after a median follow-up of 7 months. The most recent and largest case series was of 22 cases of biopsy-confirmed $\mathrm{ON}$ and included 15 cases attributed to a malabsorptive cause, 3 to a dietary cause, and 3 undetermined [8].

While there is a recognition that $\mathrm{ON}$ can be associated with both $\mathrm{EH}$ and non-EH causes, the relative frequency of each and variable clinical outcomes and histologic patterns between the 2 groups have not been reported. Thus, in this study, we defined renal histologic patterns, clinical risk factors, and patient and renal outcomes in a large cohort of biopsy-proven $\mathrm{ON}$ due to enteric and nonenteric risks, and analyzed variables predictive of adverse renal outcomes.

\section{Methods}

\section{Cohort Definition}

The study was deemed exempt by the Institutional Review Board of Mayo Clinic. The clinical data repository Advanced Cohort Explorer was used to search kidney pathology reports for $\mathrm{CaOx}$ or $\mathrm{ON}$. The search query was restricted to adult patients from 2009 to 2020 across the 3 major Mayo Clinic sites in Minnesota, Florida, and Arizona. Exclusion criteria included patients with kidney transplant prior to the date of the renal pathology re- port and patients with an ICD diagnosis of $\mathrm{PH}$. This yielded a total of 421 cases which were further reviewed to rule out $\mathrm{PH}$ and confirm a histologic diagnosis of ON based on renal pathology report and clinical records. A total of 64 cases met the inclusion and exclusion criteria, as shown in Figure 1.

\section{Data Abstraction}

Medical records were abstracted for clinical data including demographics, and the presence of hypertension (systolic BP $>140$ $\mathrm{mm} \mathrm{Hg}$, diastolic BP >90 $\mathrm{mm} \mathrm{Hg}$, or use of antihypertensive medications) or diabetes mellitus (clinically diagnosed as such by their care provider, prescribed oral hypoglycemics or insulin, or 2 fasting blood glucose measures of $140 \mathrm{mg} / \mathrm{dL}$ or greater $[7.8 \mathrm{mmol} / \mathrm{L}]$ ). The medical records were also reviewed to determine possible dietary and enteric risk factors. Presumed enteric causes included inflammatory bowel disease, celiac sprue, chronic pancreatitis (defined as $>1$ episode of acute pancreatitis), cystic fibrosis, recurrent clostridium difficile infections (defined as $>1$ infections period), small bowel resection, RYGB, and previous Whipple procedure. Other abstracted clinical variables included a history of diarrhea around the time of ON diagnosis, kidney stone events, and any gastrointestinal surgery. A dietary assessment by the evaluating nephrologist indicating excess dietary oxalate intake and any vitamin C supplementation (oral or intravenous [IV]) was recorded, as available. It should be noted that the dietary assessment of sources of oxalate was not standardized or quantified but based on the clinical judgment of the evaluating nephrologist.

Serum creatinine and eGFR (by CKD-EPI creatinine equation [9]) were collected at 3 distinct time points: $>3$ months prior to evaluation which served as baseline kidney function, at the time of evaluation and kidney biopsy, and at the last follow-up. Acute kidney injury (AKI) and CKD were defined according to the Improving Global Outcomes guidelines. Baseline CKD was determined based on eGFR $<60 \mathrm{~mL} / \mathrm{min} / 1.73 \mathrm{~m}^{2}$.

\section{Kidney Pathology Report}

All 64 renal biopsies were processed using standard techniques for light microscopy, immunofluorescence, and electron microscopy. The number of tubules that contained intraluminal or intracellular $\mathrm{CaOx}$ deposits by light and polarizing microscopy was recorded and analyzed as a binary variable $(>10)$. For 31 of the 64 cases, a descriptive term was used to describe the degree of involvement instead of a numeric value. With input from our renal pathology group, the following descriptions were grouped and extrapolated to $\mathrm{CaOx}$ tubular involvement: >10: many, numerous, significant, extensive, widespread, diffuse, $<5$ : scattered, and 5-10: moderate.

Tubular atrophy and interstitial fibrosis (IFTA) were graded on a semi-quantitative scale based on an estimate of the percentage of renal cortex affected and recorded as $1-25 \%$ (mild), $26-50 \%$ (moderate), or $>50 \%$ (severe). The presence of inflammatory interstitial cell infiltration and signs of acute tubular injury were also recorded.

\section{Statistical Analysis}

Statistical analyses were completed using JMP 9.1 (SAS, Cary, NC, USA). Fisher's exact test was used for binary data and KruskalWallis rank sum test for continuous variables. We defined adverse renal outcome as dialysis dependence or kidney transplantation at the last follow-up. Univariate and multivariate logistic regression models were built to determine odd's ratio. $p$ value $<0.05$ was defined as statistical significance. 


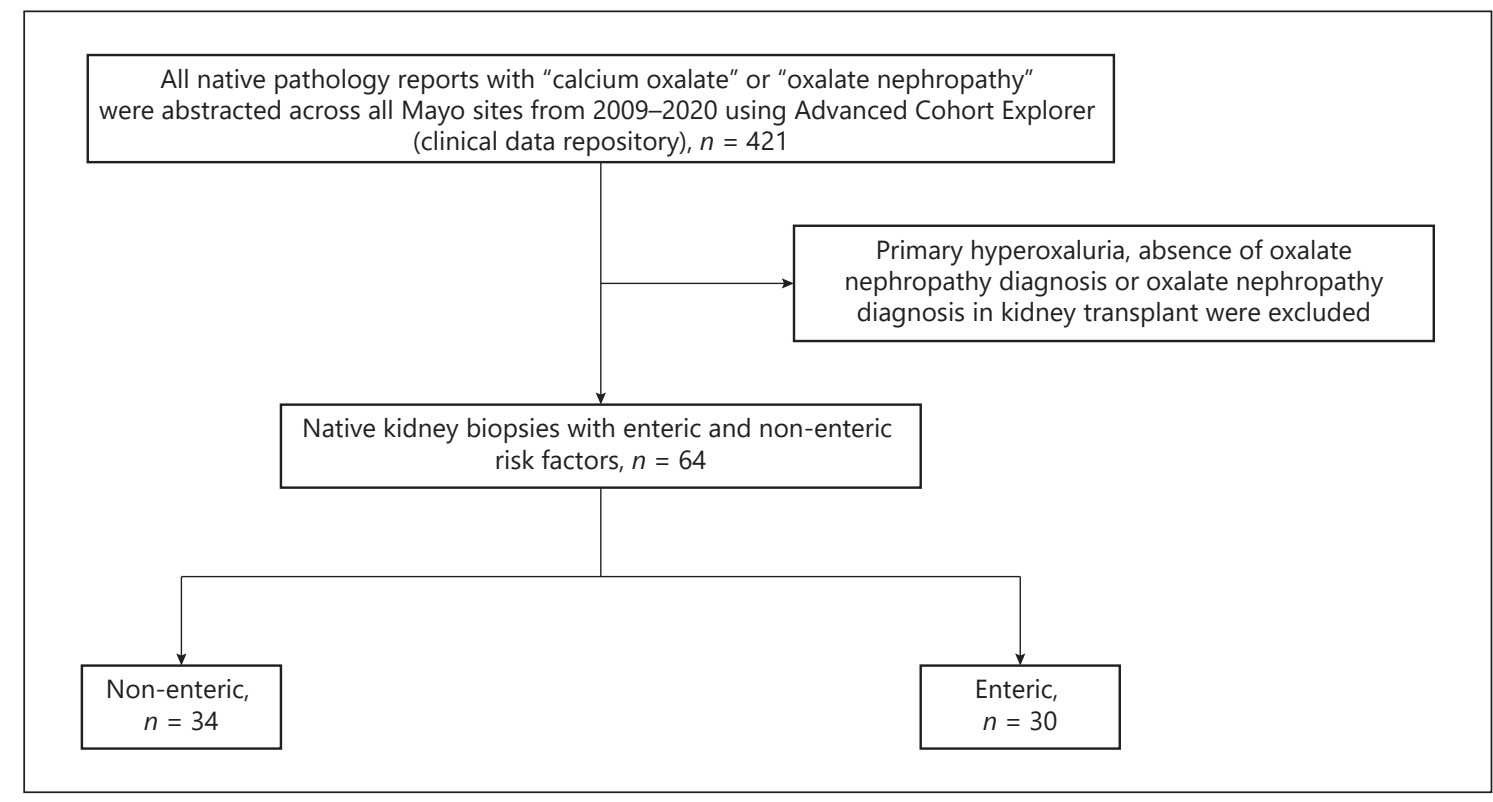

Fig. 1. Flow diagram of the identification of enteric and nonenteric $\mathrm{ON}$ cases.

\section{Results}

\section{Baseline Characteristics}

We identified a total of 64 cases of biopsy-proven ON not due to $\mathrm{PH}$. Of those, 30 cases were attributed to enteric risks and 34 due to nonenteric risks, as shown in Figure 2. Baseline characteristics are shown in Table 1. Patients in the enteric group were similar at baseline except for higher rates of baseline CKD and higher rates of diarrhea than patients with nonenteric risk factors.

\section{Risk Factors for ON}

RYGB was the most common enteric risk factor identified followed by pancreatic insufficiency, as shown in Figure 3. Among patients in the nonenteric group $(n=$ $34), 16(47.1 \%)$ did not have an apparent risk followed by an equal number of patients with vitamin $C$ intake (oral or IV, or both) $(n=7)$, and excess dietary intake of oxalate $(n=7)$ as likely triggers for ON. For patients who were identified to have ON due to vitamin C intake, 3 did not have an exact amount of vitamin $\mathrm{C}$ reported in the medical record, and for the remaining 4 patients, the amount ranged from 4,000 $\mathrm{mg}$ oral intake daily to $75,000 \mathrm{mg}$ IV 3 times a week. Ethylene glycol ingestion was noted in 3 cases, and in 1 patient, orlistat use was the identified possible risk. We next compared the subgroup of 16 patients with no apparent risk for ON compared to the nonen-

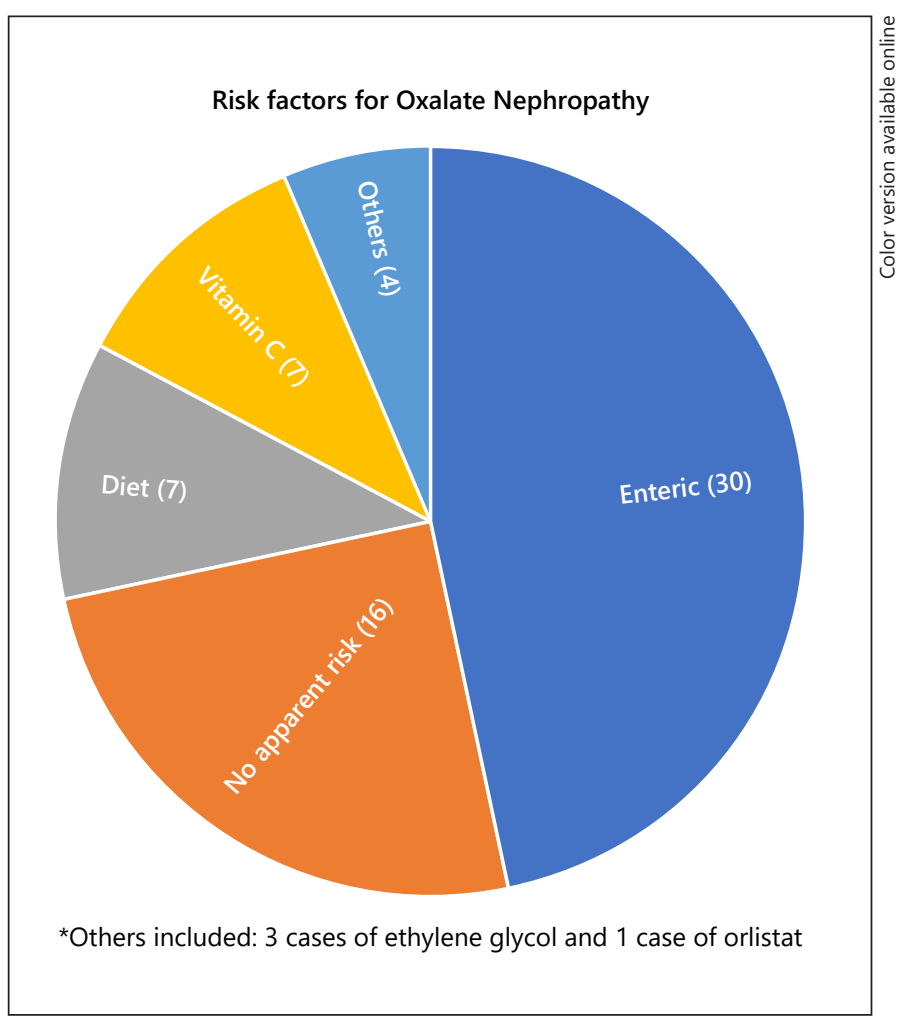

Fig. 2. Risk factors for oxalate nephropathy. 
Table 1. Baseline characteristics

\begin{tabular}{lllll}
\hline & Entire cohort (64) & Enteric (30) & Nonenteric (34) & $p$ value \\
\hline Age (mean, SD), years & $64.2,12.2$ & $65.2,8.56$ & $63.3,14.7$ & 0.50 \\
Female gender & $33,51.6$ & 18,60 & $15,44.1$ & 0.20 \\
Hypertension & $41,64.1$ & 21,70 & $20,58.8$ & 0.35 \\
Diabetes & $28,43.8$ & $14,46.7$ & $14,41.2$ & 0.66 \\
Baseline CKD & $44,68.8$ & $25,83.3$ & $19,55.9$ & 0.02 \\
Nephrolithiasis history & $12,18.8$ & 6,20 & $6,17.7$ & 0.81 \\
History of diarrhea & $21,33.3$ & $15,51.7$ & $6,17.7$ & 0.004
\end{tabular}

Values are expressed as $n, \%$, unless indicated otherwise. CKD, chronic kidney disease.



Fig. 3. Frequency of identified enteric risk factors.

teric group $(n=18)$ (Table 2). Diabetes was more common in the subgroup of patients with no apparent risk (OR 5.83, 95\% CI 1.30, 26.2, $p=0.02$ ).

\section{Clinical and Histological Patterns of ON}

At the time of ON diagnosis, all patients had an increased serum creatinine compared to baseline by a factor of 1.5 or higher, except for 1 patient with stable preexisting CKD. There were $38(60 \%)$ patients who had AKI stage III and $14(22.2 \%)$ with AKI stage II at ON diagnosis. At the time of kidney biopsy, 26 (40.6\%) required dialysis. There was no difference in AKI severity or AKI requiring dialysis between the enteric and nonenteric groups. Plasma oxalate was available for 54 patients (median $15.9 \mu \mathrm{mol} / \mathrm{L}[\min 1, \max 301 \mu \mathrm{mol} / \mathrm{L}])$ with mean creatinine of $4.94 \mathrm{mg} / \mathrm{dL}$ (SD 3.02) and mean eGFR of $14.9 \mathrm{~mL} / \mathrm{min} / .173 \mathrm{~m} 2$ (SD 9.17). Similarly, 24-h urine oxalate excretion was available in 39 patients (median 43.7 $\mathrm{mg} /$ day [min 13.2, $\max 257 \mathrm{mg} /$ day]) with mean creatinine of $4.94 \mathrm{mg} / \mathrm{dL}$ (SD 3.29). Patients with enteric risk factors tended to have a higher median plasma oxalate (18.3 vs. $10.4 \mu \mathrm{mol} / \mathrm{L}, p=0.2$ ) and urine oxalate levels (53 vs. $28.6 \mathrm{mg} / 24 \mathrm{~h}, p=0.3$ ) than patients without enteric risk factors, but the difference was not statistically significant. Among the subgroup of 16 patients with no apparent risk, $14(87.5 \%)$ had AKI stage III compared to 9 (52.9\%) patients of the nonenteric group $(p<0.05)$.

In $30(46.9 \%)$ of the patients, indirect (low appetite, nausea, vomiting, and diarrhea) or direct documentation of volume depletion was reported at the time of diagnosis, 
Table 2. Baseline characteristics between cohort with no apparent risk versus nonenteric cohort

\begin{tabular}{llll}
\hline & Unknown (16) & Nonenteric (18) & $p$ value \\
\hline Age (mean, SD), years & $64.9,9.7$ & $61.8,18.2$ & 0.50 \\
Female gender & 8,50 & $7,38.9$ & 0.51 \\
Hypertension & $10,62.5$ & $10,55.6$ & 0.68 \\
Diabetes & $10,62.5$ & $4,22.2$ & 0.02 \\
Baseline CKD & $10,62.5$ & 9,50 & 0.46 \\
Nephrolithiasis history & 4,25 & $2,11.1$ & 0.29 \\
History of diarrhea & $3,18.8$ & $3,16.7$ & 0.87 \\
\hline
\end{tabular}

Values are expressed as $n, \%$, unless indicated otherwise. CKD, chronic kidney disease.

Table 3. Histological findings $(n, \%)$

\begin{tabular}{|c|c|c|c|c|}
\hline & Entire cohort (64) & Enteric (30) & Nonenteric (34) & $p$ value \\
\hline Acute tubular injury & 59,92 & 26,87 & 33,97 & 0.11 \\
\hline Interstitial inflammation & 53,83 & 21,70 & 32,94 & 0.01 \\
\hline Tubulointerstitial fibrosis and atrophy & 61,97 & 29,97 & 32,97 & \multirow{4}{*}{0.13} \\
\hline Mild & 22,35 & 6,20 & 16,48 & \\
\hline Moderate & 22,35 & 13,43 & 9,27 & \\
\hline Severe & 17,27 & 10,33 & 7,21 & \\
\hline$>10$ tubules affected by calcium oxalate crystals & $N=61$ & 15,54 & 30,91 & 0.001 \\
\hline
\end{tabular}

and $18(28.1 \%)$ required hospitalization. The primary diagnosis for all the cases was ON. The second most common secondary diagnosis was arteriosclerosis in 11 (17.5\%), followed by diabetic glomerulosclerosis in 10 (15.9\%).

Compared to the enteric group, biopsies from the nonenteric group contained more interstitial inflammation (32 [94\%] vs. 21 [70\%], $p=0.01$ ) and a higher number of patients with $>10$ involved tubules with intratubular $\mathrm{CaOx}$ crystals (30 [91\%] vs. 15 [54\%], $p=0.003$ ) (Table 3 ). There was no difference in the overall distribution of mild, moderate, and severe IFTA between the 2 groups. However, the enteric group had $>3$ fold increased odds of moderate or severe IFTA than the nonenteric group (OR: $3.49[1.12,10.4], p=0.02)$. There were no statistically significant correlations between moderate and severe IFTA and baseline risk factors (baseline eGFR $<30$ [OR 3.5, 95\% CI $0.88,13.9], p=0.08$; diabetes (OR $2.83[0.96,8.36], p$ $=0.05)$. We also analyzed our data after exclusion of the 3 cases of ethylene glycol associated $\mathrm{ON}$, and the results remained unchanged.

Among the subgroup of patients with no apparent risk, $11(68.8 \%)$ had moderate-severe IFTA compared to 5 (29.4\%) of patients in the nonenteric group $(p=0.02)$. All 16 patients had $>10$ involved tubules with intratubular
$\mathrm{CaOx}$ crystals. There was no difference in plasma oxalate concentration or urine oxalate excretion, or in the rates of acute tubular injury or interstitial inflammation between the no apparent risk cohort compared to the nonenteric group. Treatment approach was variable and included IV hydration (in all patients), oral calcium supplementation for oxalate binding, pancreatic enzyme supplementation $(n=1)$, bile acid binding resin $(n=1)$, and corticosteroid course $(n=13$ [ 1 patient in enteric group and 12 in nonenteric group]); 51 (89\%) received counseling for low-fat and low-oxalate diet.

\section{Clinical Outcomes at the Last Follow-Up}

After median follow-up time of 10 months after ON diagnosis, most patients $(n=36)$ had an eGFR $<30 \mathrm{~mL} /$ $\mathrm{min} / 1.73 \mathrm{~m}^{2}, 25$ were dialysis dependent, and 7 had received a kidney transplant. Another 4 (6.2\%) patients recovered kidney function, 10 (15.6\%) remained on dialysis, 8 (17.1\%) underwent kidney transplant, 24 (37.5\%) had CKD stage 4, and 19 (29.6\%) had CKD stage 3. There was no significant difference in renal outcome between the enteric and nonenteric groups, as shown in Table 4. A total of $20(32 \%)$ patients had died at the last follow-up with no difference in the 2 groups. There was no difference in outcome between the no apparent risk cohort and 
Table 4. Kidney function at the last followup $(n, \%)$

\begin{tabular}{lllll}
\hline & Entire cohort (64) & Enteric (30) & Nonenteric (34) & $p$ value \\
\hline Dialysis dependent & 25,39 & 10,33 & 15,44 & 0.38 \\
Received kidney transplant & 7,11 & $1,3.3$ & 6,18 & 0.07 \\
eGFR $<15 \mathrm{~mL} / \mathrm{min} / 1.73 \mathrm{~m}^{2}$ & 20,38 & 5,26 & 15,44 & \\
eGFR $15-29 \mathrm{~mL} / \mathrm{min} / 1.73 \mathrm{~m}^{2}$ & 16,30 & 8,42 & 8,24 & \\
eGFR $30-59 \mathrm{~mL} / \mathrm{min} / 1.73 \mathrm{~m}^{2}$ & 13,25 & 5,26 & 8,24 & \\
eGFR $60-89 \mathrm{~mL} / \mathrm{min} / 1.73 \mathrm{~m}^{2}$ & $2,3.8$ & $1,5.3$ & $1,2.9$ & \\
eGFR $\geq 90 \mathrm{~mL} / \mathrm{min} / 1.73 \mathrm{~m}^{2}$ & $2,3.8$ & 0 & $2,5.9$ & \\
\hline
\end{tabular}

the nonenteric cohort, except that kidney transplant occurred in 5 of the no apparent risk cohort (5 [31.3\%] vs. $1[5.56 \%]$ in the nonenteric cohort $(p=0.04)$. Of note, 32 $(62.6 \%)$ patients who received counseling for low-fat and low-oxalate diet did not require dialysis or transplant at the last follow-up compared to those who did not receive dietary counseling ( $n=4[30.8 \%], p=0.038)$.

We next assessed factors associated with an increased risk of adverse renal outcome $(n=28)$ across the entire group. On univariate analysis baseline CKD (OR 3.29, $95 \%$ CI $[1.02,10.6], p=0.05)$, $>10$ tubules involved with $\mathrm{CaOx}$ crystals (OR 4.95, 95\% CI $[1.24,19.8], p=0.02$ ), and AKI requiring dialysis at the time of diagnosis were significant (OR 22.0, 95\% CI [5.95, 81.4], $p<0.001$ ). On multivariate analysis, only AKI requiring dialysis was statistically significantly associated with adverse renal outcome. These findings were similar when the outcome of interest included adverse renal outcome and/or mortality at the last follow-up. The presence of interstitial inflammation, enteric versus nonenteric risk factors, or degree of IFTA and presence of inflammation were not associated with renal outcome.

\section{Discussion}

This is the largest case cohort study of biopsy-proven ON in native kidneys comparing 2 cohorts of patients: those with well-identified enteric risk factors and those without. We show unique differences in baseline CKD and renal histologic patterns in the enteric and nonenteric risk cohorts and found that AKI requiring dialysis at the time of diagnosis was the single most important predictor of adverse renal outcome. In the subgroup of patients with no apparent risk diabetes, severe AKI and moderate-to-severe tubulointerstitial fibrosis were common.

In the first case series of ON due to RYGB [6], 7 of the 11 patients had moderate-to-severe IFTA which is consistent with our observation that the enteric risk group had more severe IFTA than the nonenteric risk group, and IFTA was associated with a higher rate of baseline CKD in the enteric risk group in our study. In a case series of chronic pancreatitis patients with ON [7], acute tubular injury was the most common finding, but the degree of intratubular $\mathrm{CaOx}$ involvement and IFTA were not reported. Our findings demonstrate that the degree of intratubular $\mathrm{CaOx}$ involvement and associated interstitial inflammation is increased in the nonenteric risk group compared to the enteric group. This contrasts with the case series of Nasr et al. [6] in which interstitial inflammation was universal and the median number of $\mathrm{CaOx}$ deposits was 31.5. This could possibly be explained by the longer latency period from RYGB surgery to ON diagnosis in our study (median 8 ; range: $\min 1$ and max 40 years) than that in the Nasr case series (median 12; min 4 months and max 96 months). This observation suggests that $\mathrm{ON}$ may evolve as chronic tubulointerstitial inflammation with CKD progression. It can therefore be hypothesized that increased awareness and early detection of ON may influence renal outcomes. Ongoing study of enteric and nonenteric cases of $\mathrm{ON}$ are needed to help explain variability in intratubular $\mathrm{CaOx}$ involvement and its associated inflammatory blueprint.

In the nonenteric group, most patients $(n=16)$ did not have an identifiable trigger for ON. An equal number of patients had increased dietary intake of oxalate $(n=7)$ or oxalate precursor in the form of vitamin C $(n=7)$. In 2018 , a systematic review of case reports and case series of all ON cases in native and transplanted patients reported that a known enteric risk was most frequent followed by increased intake of dietary or supplemental oxalate [10]. In our study, 16 patients had no apparent risk, 6 of whom underwent genetic testing for $\mathrm{PH}$ which was negative. This raises the question regarding current gaps in evaluating triggers for $\mathrm{ON}$ cases. We hypothesize that $\mathrm{ON}$ develops as a multi-hit process that culminates in a high tubular concentration of oxalate, leading to $\mathrm{CaOx}$ crystals followed by adhesion to cells, transcytosis to the interstitium, and an 
inflammatory response. In animal models, hyperoxlauria and intrarenal $\mathrm{CaOx}$ crystals have been shown to activate the nucleotide-binding domain leucine-rich repeat inflammasome responsible for the activation of inflammatory cytokines such as IL-1B and IL-18, and for propagating interstitial fibrosis and tubular atrophy [11-13]. Several stimuli have been shown to trigger the assembly of the nucleotide-binding domain leucine-rich repeat inflammasome including ion flux (e.g., low intracellular potassium as might occur in the setting of acidemia), oxidative stress, mitochondrial dysfunction (direct or drug induced), or ineffective phagocytosis of large crystals (including cholesterol crystals and calcium) [14]. Thus, in addition to increased enteric oxalate absorption or reduced metabolism, other factors such as volume depletion, acute or chronic inflammatory states, concurrent illness, acid base imbalances, and medications that impact lysosomal or mitochondrial function may all contribute to the development of ON. Diabetes was more prevalent in our cohort with no apparent ON risk than in the nonenteric group. Similarly, markers of volume depletion were present in $47 \%$ of patients. In a study of over 3,000 patients with CKD, diabetes was an independent predictor of increased urinary oxalate excretion, which is a critical step in the development of $\mathrm{ON}$ [15]. There is also evidence to suggest increased endogenous production of oxalate precursors (which also promote oxidative stress) in patients with diabetes [16]. DM is also an established risk factor for both CKD as well as AKI. Thus, diabetes may contribute in multiple ways to a multihit process that culminates in ON. In the largest prior ON case series $(n=21)$, only 3 patients did not have an identified ON risk (14\%) which is lower than our study $(n=16$, $25 \%$ ) [8]. It is of interest that 2 of the 3 patients without apparent ON risk in this previous series (66.7\%) had diabetes and stage 3-4 CKD. In our no apparent risk subgroup, 10 (62.5\%) had DM and 10 (62.5\%) had CKD which mirrors the results in the study by Buysschaert et al. [8] In that same study, all 3 patients with no apparent risk for $\mathrm{ON}$ had a workup for malabsorption which was negative. In our study, only 7 of the 16 patients with no apparent risk were referred to gastroenterology for evaluation of an enteric risk, and none were identified.

We identified high rates of CKD stage 4 or greater (56\%) and a $32 \%$ mortality at the last follow-up. This is consistent with other published work including a case series of $22 \mathrm{ON}$ patients that reported $30 \%$ mortality and $52 \%$ with kidney failure [8], and a systematic review by Lumlertgul et al. [10] that reported dialysis-dependent kidney failure in $55 \%$ of biopsy-confirmed ON cases. In our study, AKI requiring dialysis at the time of $\mathrm{ON}$ diag-

Oxalate Nephropathy in Patients with

Enteric and Nonenteric Risk Factors nosis was the most significant risk factor for long-term dialysis or needing kidney transplant. Thus, baseline kidney function is an important prognostic factor as also reported by Buysschaert et al. [8].

There are several limitations that warrant discussion. The study was retrospective and spanned over a decade which may have contributed to the variability in treatment and diagnostic approach. The sample size was relatively small which limited our ability to assess differences and outcomes within subgroups of ON cases. However, this remains the largest cohort study of $\mathrm{ON}$ to date. The underlying cause in our enteric versus nonenteric groups may have had some degree of overlap due to the unclear nature of the diagnosis in some. Cases ascribed to dietary risk factors could not be confirmed due to the lack of the standardized approach to estimate dietary oxalate content. Long-term outcomes of those who underwent kidney transplant were not ascertained. Furthermore, the diagnosis of ON was based on the renal pathologist's final impression informed by histological findings and confirmed by the clinical nephrologist. Finally, a prespecified $\mathrm{CaOx}$ crystal to glomerulus ratio was not used to define the $\mathrm{ON}$ diagnosis.

\section{Conclusion}

This is the largest single-center study to date of ON due to secondary risk factors. Our data demonstrate that RYGB remains the most common risk followed by a cohort of patients with no apparent cause, highlighting a need to better understand the pathophysiology of $\mathrm{ON}$. Nonenteric cases of ON appear to have more interstitial inflammation, corresponding with a greater number of intratubular $\mathrm{CaOx}$ crystals, while enteric cases demonstrate more evidence for $\mathrm{CKD}$ and a greater amount of moderate and severe IFTA. Overall, severity of AKI is the most important prognostic factor impacting long-term renal outcome.

\section{Acknowledgements}

The authors acknowledge the contributions of Ali Mahmoud and Marcelino Rivera who abstracted data for 4 of the cases included in this study.

\section{Statement of Ethics}

Study approval statement: Mayo Clinic Institutional Review Board deemed the study not to require ethics approval. Consent to participate statement: Written informed consent was not required. 


\section{Conflict of Interest Statement}

Mira T. Keddis discloses employment from Mayo Clinic, consultancy agreement from ALLENA Pharmaceuticals, and Honoraria received from the Chronic Liver Disease Foundation. John C. Lieske discloses receiving research grants from Alnylam, Dicerna, Allena, and OxThera. He also serves on the scientific advisory board for Alnylam, Dicerna, Orfan, Synlogic, and Novobiome. All other authors have no conflicts of interest to declare.

\section{Funding Sources}

No funding was utilized for this study.

\section{Author Contributions}

S.R., E.B., and M.A. provided substantial contribution to the acquisition, analysis, and interpretation of the work. S.R. drafted the manuscript. J.L provided important contribution to conception, analysis, and interpretation and critical revision of the manuscript. M.R. provided critical input into analysis and revision of the manuscript. M.K. provided concept, design, and assisted with data acquisition, and completed analysis and critical revision of the manuscript. All authors agreed to be accountable for all aspects of the work and approved the final version.

\section{Data Availability Statement}

The dataset used for analysis of the study is available from the corresponding author upon reasonable request.

\section{References}

1 Hylander E, Jarnum S, Jensen HJ, Thale M. Enteric hyperoxaluria: dependence on small intestinal resection, colectomy, and steatorrhoea in chronic inflammatory bowel disease. Scand J Gastroenterol. 1978;13(5):577-88.

2 Asplin JR. The management of patients with enteric hyperoxaluria. Urolithiasis. 2016 Feb; 44(1):33-43.

3 Knauf F, Brewer JR, Flavell RA. Immunity, microbiota and kidney disease. Nat Rev Nephrol. 2019 May;15(5):263-74.

4 Cryer PE, Garber AJ, Hoffsten P, Lucas B, Wise L. Renal failure after small intestinal bypass for obesity. Arch Intern Med. 1975 Dec; 135(12):1610-2.

5 Mole DR, Tomson CR, Mortensen N, Winearls CG. Renal complications of jejunoileal bypass for obesity. QJM. 2001 Feb;94(2): 69-77.

6 Nasr SH, D'Agati VD, Said SM, Stokes MB, Largoza MV, Radhakrishnan J, et al. Oxalate nephropathy complicating Roux-en-Y gastric bypass: an underrecognized cause of irreversible renal failure. Clin J Am Soc Nephrol. 2008 Nov;3(6):1676-83.
7 Cartery C, Faguer S, Karras A, Cointault O, Buscail L, Modesto A, et al. Oxalate nephropathy associated with chronic pancreatitis. Clin J Am Soc Nephrol. 2011 Aug;6(8):1895902.

8 Buysschaert B, Aydin S, Morelle J, Gillion V, Jadoul M, Demoulin N. Etiologies, clinical features, and outcome of oxalate nephropathy. Kidney Int Rep. 2020 Sep;5(9):1503-9.

9 Inker LA, Schmid CH, Tighiouart H, Eckfeldt JH, Feldman HI, Greene T, et al. Estimating glomerular filtration rate from serum creatinine and cystatin C. N Engl J Med. 2012 Jul 5; 367(1):20-9.

10 Lumlertgul N, Siribamrungwong M, Jaber BL, Susantitaphong P. Secondary oxalate nephropathy: a systematic review. Kidney Int Rep. 2018 Nov;3(6):1363-72.

11 Knauf F, Asplin JR, Granja I, Schmidt IM, Moeckel GW, David RJ, et al. NALP3-mediated inflammation is a principal cause of progressive renal failure in oxalate nephropathy. Kidney Int. 2013 Nov;84(5):895-901.
12 Mulay SR, Kulkarni OP, Rupanagudi KV, Migliorini A, Darisipudi MN, Vilaysane A, et al. Calcium oxalate crystals induce renal inflammation by NLRP3-mediated IL- $1 \beta$ secretion. J Clin Invest. 2013 Jan;123(1):236-46.

13 Ludwig-Portugall I, Bartok E, Dhana E, Evers BD, Primiano MJ, Hall JP, et al. An NLRP3specific inflammasome inhibitor attenuates crystal-induced kidney fibrosis in mice. Kidney Int. 2016 Sep;90(3):525-39.

14 Kelley N, Jeltema D, Duan Y, He Y. The NLRP3 inflammasome: an overview of mechanisms of activation and regulation. Int $\mathrm{J} \mathrm{Mol}$ Sci. 2019 Jul 6;20(13):3328.

15 Waikar SS, Srivastava A, Palsson R, Shafi T, Hsu CY, Sharma K, et al. Association of urinary oxalate excretion with the risk of chronic kidney disease progression. JAMA Intern Med. 2019 Apr 1;179(4):542-51.

16 Wang XJ, Ma SB, Liu ZF, Li H, Gao WY. Elevated levels of $a$-dicarbonyl compounds in the plasma of type II diabetics and their relevance with diabetic nephropathy. J Chromatogr B Analyt Technol Biomed Life Sci. 2019 Feb 1;1106:19-25. 